SHORT REPORT

\title{
Sequential constriction of upper airway and vocal cords in sleep apnoea of multiple system atrophy: low field magnetic resonance fluoroscopic study
}

\author{
M Hirayama, H Fukatsu, H Watanabe, Y Koike, A Noda, H Ito, R Kobayashi, G Sobue
}

J Neurol Neurosurg Psychiatry 2003;74:642-645

Low field magnetic resonance fluoroscopy was used to clarify temporal and spatial features of airway obstruction in sleep apnoea syndrome (SAS) in multiple system atrophy (MSA), as well as in obstructive SAS (OSAS). 20 patients with OSAS with severe obesity (mean (SD) age 66 (10) years; 16 men, 4 women) and 6 patients with SAS related to probable MSA (60 (9) years; 4 men, 2 women) were studied. In the OSAS group, body mass index, apnoea index, and desaturation index were significantly higher than in the MSA group. In OSAS, simultaneous obstruction extended from the retropalatal pharynx to the retroglossal during sleep on low field magnetic resonance fluoroscopy. In MSA, obstruction of upper airway followed a similar distribution, but obstruction of vocal cords followed upper airway obstruction. In contrast to OSAS, sequentially acting neural mechanisms are suspected in SAS with MSA.

O bstructive sleep apnoea syndrome (OSAS), commonly associated with obesity, causes obstruction of the upper airway. OSAS is considered to be a risk factor for cardiovascular disease and for traffic accidents. In patients with multiple system atrophy (MSA), a sleep apnoea syndrome (SAS) occurs frequently, ${ }^{1}$ occasionally causing sudden death. ${ }^{2}$ However, duration of apnoea generally is shorter and $\mathrm{O}_{2}$ desaturation is milder in MSA than in OSAS. ${ }^{2}$ The pathophysiology of SAS in MSA is incompletely understood..$^{3-6}$

High speed computed tomography, magnetic resonance (MR) imaging, and laryngoscopy have been used to assess the upper airway in SAS. ${ }^{7-9}$ However, high speed computed tomography does not permit a sufficiently long period of monitoring of the upper airway during sleep. Laryngoscopy also cannot be used without anaesthesia and is difficult to use during natural sleep because of the discomfort it causes.

To clarify the pathophysiology of SAS in natural sleep, we have developed a new method to monitor the upper airway using low field MR fluoroscopy, which provided useful evaluation of SAS. ${ }^{10}{ }^{11}$ In the present study we compared the temporal and spatial profile of airway obstruction in sleep apnoea in MSA with that in OSAS.

\section{PATIENTS AND METHODS}

Twenty patients with OSAS with severe obesity (mean (SD) age 66 (10) years; 16 men, 4 women) and six patients with SAS related to probable MSA $^{12}$ (60 (9) years; 4 men, 2 women) were studied (table 1). In these patients OSAS or MSA was diagnosed, and they were treated at Nagoya University Hospital. Patients in both groups all were evaluated by polysomnography and fulfilled diagnostic criteria for SAS. Subjects gave informed consent in advance.

Most patients underwent MR fluoroscopic assessment during the day (1:30 to $3: 30 \mathrm{pm}$ ) or around midnight ( $11 \mathrm{pm}$ to $2 \mathrm{am}$ ) in natural sleep in the supine position. Images were recorded for at least 60 minutes, and evidence of sleep related obstructive phenomena was sought in all patients during MR imaging. Since it is difficult to record an electroencephalogram during MR imaging, we identified the sleep state by inspection and presence of snoring or stridor.

An open gantry MR imager (Toshiba, Tokyo, Japan) with a $0.35 \mathrm{~T}$ magnet was used. A solenoid belt coil was placed

Abbreviations: $B M I$, body mass index; MR, magnetic resonance; MSA, multiple system atrophy; OSAS, obstructive sleep apnoea syndrome; SAS, sleep apnoea syndrome

Table 1 Clinical details and magnetic resonance imaging (MRI) findings in patients with multiple system atrophy (MSA) and with obstructive sleep apnoea syndrome (OSAS)

\begin{tabular}{|c|c|c|c|c|c|c|c|c|c|c|}
\hline \multirow[b]{2}{*}{ Patient } & \multirow[b]{2}{*}{$\begin{array}{l}\text { Age } \\
\text { (years) }\end{array}$} & \multirow[b]{2}{*}{ Sex } & \multirow[b]{2}{*}{$\begin{array}{l}\text { Disease } \\
\text { type }\end{array}$} & \multirow[b]{2}{*}{$\begin{array}{l}\mathrm{BMI} \\
\left(\mathrm{kg} / \mathrm{m}^{2}\right)\end{array}$} & \multirow[b]{2}{*}{$\begin{array}{l}\text { Apnoea } \\
\text { index }(/ \mathrm{h})\end{array}$} & \multirow[b]{2}{*}{$\begin{array}{l}\text { Desaturation } \\
\text { index }(/ \mathrm{h})\end{array}$} & \multirow[b]{2}{*}{$\begin{array}{l}\text { Lowest } \mathrm{O}_{2} \\
\text { saturation (\%) }\end{array}$} & \multicolumn{3}{|l|}{ MRI findings } \\
\hline & & & & & & & & $\begin{array}{l}\text { Tongue } \\
\text { atrophy }\end{array}$ & $\begin{array}{l}\text { Upper airway } \\
\text { obstruction }\end{array}$ & $\begin{array}{l}\text { Vocal cord } \\
\text { obstruction }\end{array}$ \\
\hline \multicolumn{11}{|l|}{ MSA } \\
\hline 1 & 50 & $\mathrm{~F}$ & C & 29.7 & 28 & 20 & 73 & Complete & Complete & Absent \\
\hline 2 & 73 & $M$ & C & 17.8 & 17 & 4 & 89 & Complete & Complete & Present \\
\hline 3 & 64 & $M$ & $\mathrm{C}$ & 29.4 & 39 & 29 & 65 & Complete & Complete & Absent \\
\hline 4 & 56 & $M$ & C & 25.4 & 25 & 10 & 85 & Complete & Complete & Absent \\
\hline 5 & 54 & $\mathrm{~F}$ & $P$ & 17.8 & 12 & 1 & 95 & Incomplete & Incomplete & Present \\
\hline 6 & 65 & $M$ & $\mathrm{C}$ & 19.6 & 14 & 10 & 75 & Incomplete & Incomplete & Absent \\
\hline OSAS $(n=20)$ & & $\begin{array}{l}M=16 \\
F=4\end{array}$ & & $32(5.5)$ & 44 (12) & $28(15)$ & 70 (10) & $\begin{array}{l}\text { Incomplete to } \\
\text { complete }\end{array}$ & Absent & Absent \\
\hline
\end{tabular}

OSAS data are mean (SD). BMI, body mass index; $C$, cerebellar MSA; F, female; $M$, male; $P$, parkinsonian MSA. Disease types $C$ and $P$ are based on the diagnostic criteria of Gilman et al. ${ }^{12}$ 

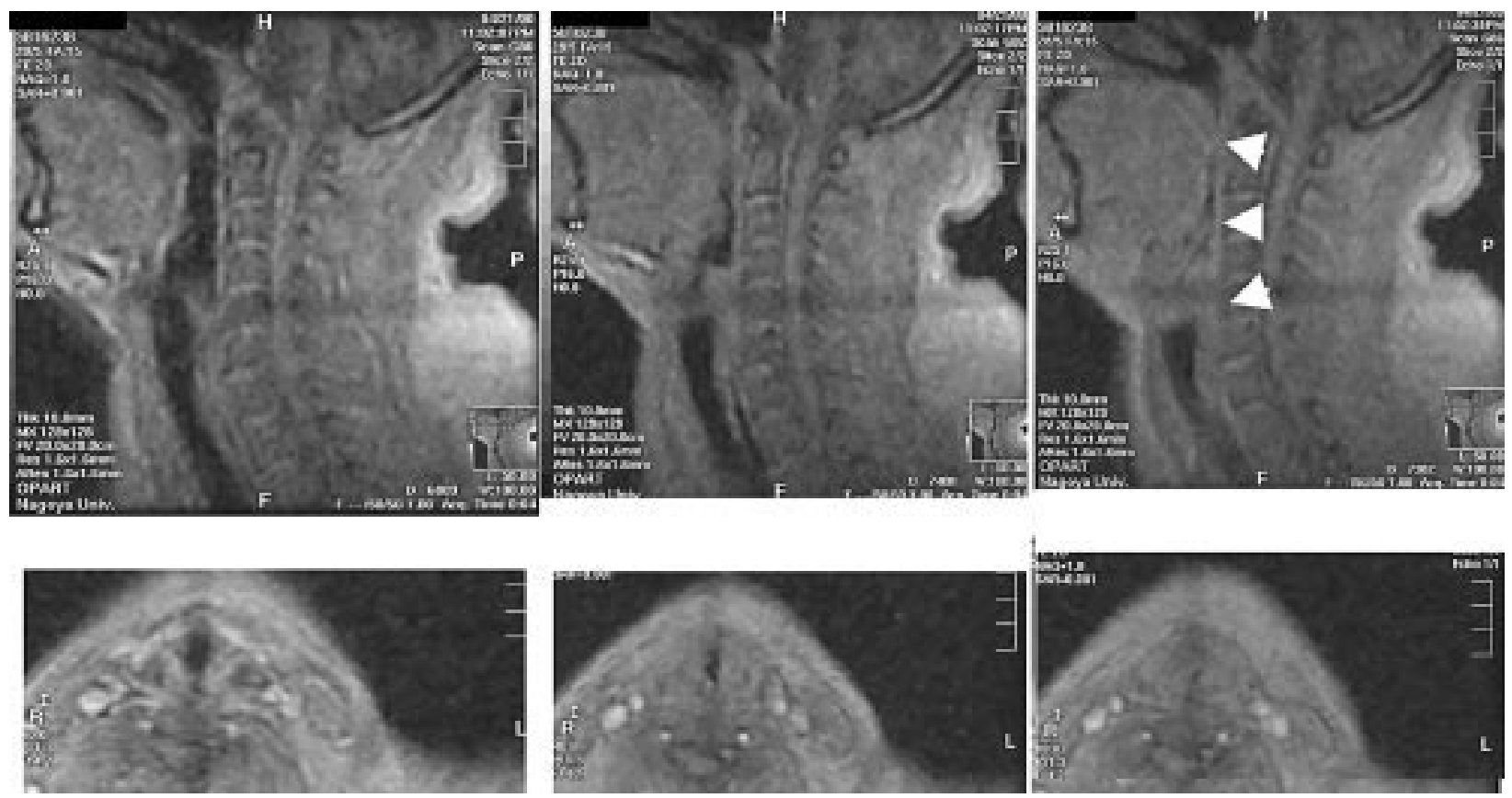

Figure 1 Low field magnetic resonance imaging in the upper airway and vocal cords in a patient with multiple system atrophy (MSA) with obesity. Snoring or stridor occurred following upper airway obstruction. At the same time the glottis narrowed. After upper airway obstruction progressed to completion, the glottis occluded completely (arrowheads).

around the patient's neck. MR fluoroscopy was performed using a fast spin echo-type sequence with repetition time of $28 \mathrm{~ms}$, echo time of $5 \mathrm{~ms}$, and flip angle of $15^{\circ}$. Slice thickness was $10 \mathrm{~mm}$, and a $23 \mathrm{~cm}$ field of view was imaged continuously with a $128 \times 128$ matrix. The midsagittal plane and the transaxial plane at the soft palate level usually were selected for monitoring. Real time images were monitored at 0.9 second intervals. The difference in the parameter of sleep apnoea between OSAS an MSA groups was statistically analysed by Student's $t$ test. To identify the narrowing of the vocal cord, we assessed the maximum diameter of the vocal cord image on MR imaging that adjusted on the plane of the vocal cord, and we determined the presence of narrowing of the vocal cord when the diameter was less than half that in the awake state.

\section{RESULTS}

In the OSAS group, body mass index (BMI), apnoea index, and desaturation index were significantly higher than in the MSA group (OSAS $v$ MSA: BMI 32 (5.5) $v 23$ (5.6), p < 0.01;
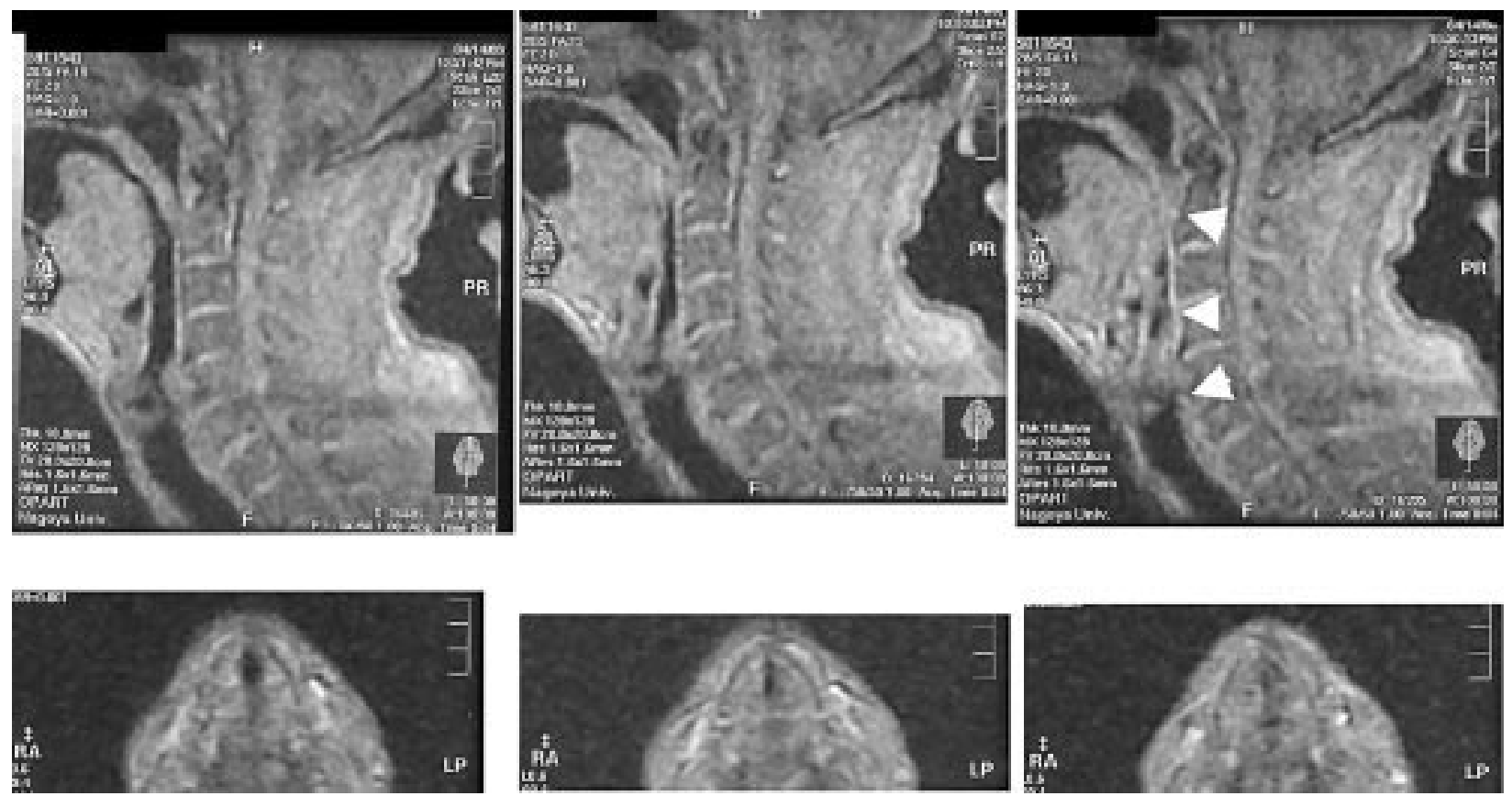

Figure 2 Low field MR imaging in the upper airway and vocal cords in MSA in a patient with atrophic tongue. This patient had severe atrophy of the tongue and laryngopharyngeal muscles. Snoring or stridor occurred from upper airway obstructions; at the same time, the glottis narrowed. Upper airway and vocal cords both were completely occluded (arrowheads). 

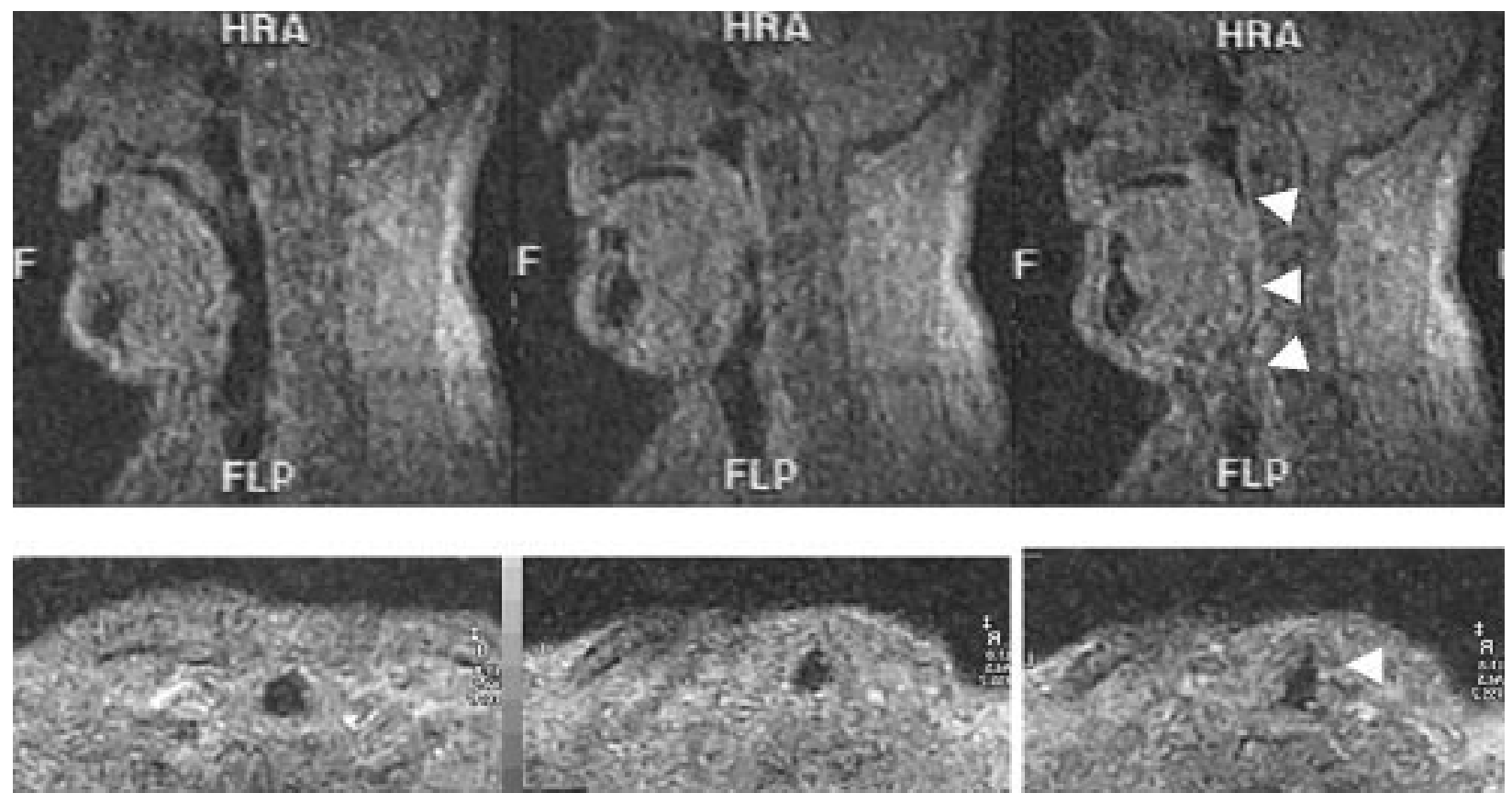

Figure 3 Low field MR imaging in the upper airway and vocal cords in a patient with obstructive sleep apnoea syndrome. The upper airway was occluded, but the glottis was not (arrowheads).

apnoea index 44 (12) $v 22$ (10), p < 0.01; desaturation index 28 (15) $v 12(10), \mathrm{p}<0.05$; table 1). In patients with MSA, no upper airway obstruction occurred during wakefulness, but the vocal cord of the airway was slightly narrowed. In SAS episodes, snoring or stridor was notable following upper airway narrowing, when the vocal cord was additionally narrowed. Upper airway stenosis occurred at the palatopharyngeal level initially and then extended to the glossopharyngeal level, progressing to complete obstruction; then the glottis was occluded completely. This sequential obstructive pattern in the upper airway extending to the vocal cords was observed in all SAS episodes in six patients with MSA, even in those with atrophy of the tongue (table 1 , fig 1 , fig 2 ). This suggested that obesity with increased BMI did not contribute to this phenomenon. Obstruction of the vocal cord alone without upper airway obstruction was not observed in any patient.

In the OSAS group, no upper airway obstruction occurred during wakefulness, but repeated episodes of upper airway obstruction were observed in natural sleep in all 20 patients. In these sleep apnoea episodes, complete obstruction occurred in a descending sequence in the upper airway, as in MSA; however, vocal cord obstruction was not observed in any patient. This obstruction pattern was similar in all 20 patients with OSAS (table 1, fig 3).

\section{DISCUSSION}

Visualising occlusive events in the upper airway in OSAS and sleep apnoea in MSA is difficult. Use of a fibreoptic laryngoscope easily displays the upper airway but cannot be used during natural sleep because of discomfort. ${ }^{7}$ In addition, since this instrument cannot visualise areas distal to a site of obstruction, sequential assessment of airway obstruction in time and space is not possible. Upper airway monitoring using low field MR fluoroscopy is a new method that can sequentially analyse both the upper airway and the vocal cord simultaneously during natural sleep. This is the first report of spatial and temporal profiles of the upper airway and vocal cord during natural sleep in patients with MSA.

In OSAS, upper airway obstruction was found at the level of palatopharynx, with extension to the glossopharynx but without vocal cord obstruction. In MSA, however, both the upper airway and the vocal cords were obstructed sequentially. Progress has occurred in understanding the mechanisms of vocal cord obstruction in MSA. Vocal cord abductor paralysis (Gerhardt syndrome) has been suspected from pathological and electrophysiological viewpoints. ${ }^{3-6}$ 13-16 $^{16}$ Selective neurogenic atrophy of the posterior cricoarytenoid muscle has been documented by postmortem observations in MSA. ${ }^{13}{ }^{16}$ Electromyographic evidence of a neurological abnormality also was found in the posterior cricoarytenoid muscle. ${ }^{37}$ Since this muscle is the sole abductor of the vocal cords, airway stenosis result from paralysis of this muscle. Even during wakefulness, partial vocal cord paralysis is observed in patients with MSA, as in the present study. However, most recent electrophysiological studies have shown that the cause of stridor is hyperactivity (dystonia) rather than paralysis of the vocal cord abductors, ${ }^{56}$ suggesting supranuclear neurological dysfunction as the cause of glottic obstruction. These results may differ because of the stage of disease in each study. Vocal cord stenosis was seen without vocal cord abductor paralysis.

Another important question concerns why upper airway obstruction and vocal cord obstruction occur sequentially in patients with MSA. In obese patients with MSA, upper airway obstruction may partially account for sleep apnoea by the same mechanism as in OSAS patients. ${ }^{17}$ However, obstruction of the upper airway and at the vocal cords occurred in a patient with atrophy of the tongue and laryngopharyngeal muscles, suggesting another mechanism of upper airway obstruction distinct from OSAS. Vocal cord obstruction always occurred following upper airway obstruction in all patients with MSA. Although details of innervation of upper airway muscles from the brain stem are not entirely known in humans, motor centres in the nucleus ambiguus innervate both laryngopharyngeal and vocal cord muscles in monkey. ${ }^{18}$ We suspect that neural events resembling those causing vocal cord obstruction may occur in upper airway muscles. The larynx can easily be pulled inwards and downwards, narrowed by negative intratracheal pressure causing inspiratory collapse in MSA. ${ }^{7}$ Once upper airway narrowing occurs, laryngeal and vocal cord stenosis can occur easily as an exacerbation. ${ }^{19}$ A highly synchronised neural mechanism appears to be important for sleep apnoea in MSA, which may involve excessive muscle contraction as in dystonia-like phenomena. Certain laryngeal stimuli 
such as contact with water or negative pressure readily induce glottic stenosis. ${ }^{19}$ Such stimulation induced immediate laryngeal closure and inspiratory flow limitation developed five minutes after the stimulation. ${ }^{19}$ Furthermore, persistent dystonia-like electromyographic activity of the cricopharyngeal muscle was seen in MSA during the hypopharyngeal phase of deglutition, ${ }^{20}$ supporting the view that abnormal reflex of laryngeal closure rather than paralysis causes upper airway obstruction and vocal cord obstruction at that level in MSA.

These results may suggest the following hypothesis that sleep apnoea in MSA occasionally causes sudden death. During sleep, hypotonia at the base of the throat causes obstruction of the upper airway, with extremely loud snoring and laboured breathing leading to apnoea in patients with OSAS. Some stimulations (hypoxaemia and hypercapnia causing apnoea) result in an arousal response, eliminating the hypotonia maintaining the upper airway. As for sleep apnoea in MSA, if the upper airway is obstructed, it stimulates more excessive upper airway and vocal cord closure. ${ }^{19}$ Hypoxia and hypercapnia cause an arousal response, which may cause a more excessive muscle contraction and lead to a dystonia-like reaction. Consequently, more prolonged apnoea induces cardiac arrhythmia.

\section{Authors' affiliations}

M Hirayama, H Fukatsu, H Watanabe, Y Koike, A Noda, H Ito, R Kobayashi, G Sobue, Department of Neurology, Nagoya University School of Medicine, Nagoya, Japan

Correspondence to: Professor G Sobue, Department of Neurology, Nagoya University School of Medicine, 65 Tsurumai-cho Showa-ku, Nagoya 466-8550 Japan; sobueg@med.nagoya-u.ac.jp

Received 17 October 2002

In revised form 4 December 2002

Accepted 11 December 2002

\section{REFERENCES}

1 Bannister R, Oppenheimer DR. Degenerative diseases of the nervous system associated with autonomic failure. Brain 1972;95:457-74.
2 Munschauer FE, Loh $L$, Bannister $R$, et al. Abnormal respiration and sudden death during sleep in multiple system atrophy with autonomic failure. Neurology 1990;40:677-9.

3 Guindi GM, Bannister R, Gibson WPR, et al. Laryngeal electromyography in multiple system atrophy with autonomic failure. $J$ Neurol Neurosurg Psychiatry 1981;44:49-53.

4 Koike $Y$, Hirayama M, leda T, et al. Polysomnographic differences between Shy-Drager syndrome and sleep apnea syndrome. Jiritsu Shinkei 1998;35:461-8

5 Merlo IM, Occhini A, Pacchetti C, et al. Not paralysis, but dystonia causes stridor in multiple system atrophy. Neurology 2002;58:649-52.

6 Simpson DM, Kaufmann H, Sanders I, et al. Laryngeal dystonia in multiple system atrophy. Muscle Nerve 1992:5:1213-5.

7 Isozaki E, Naito A, Horiguchi S, et al. Early diagnosis and stage classification of vocal cord abductor paralysis in patients with multiple system atrophy. J Neurol Neurosurg Psychiatry 1996;60:399-402.

8 Suto $Y$, Inove Y. Sleep apnea syndrome: examination of pharyngeal obstruction with high speed MR and polysomnography. Acta Radiol 1996;37:315-20.

9 Bohlman ME, Haponik EF, Smith PL et al. CT demonstration of pharyngeal narrowing in adult obstructive sleep apnea. Am J Roentgenol 1983;140:543-8.

10 Yoshida K, Fukatsu H, Ando Y, et al. Evaluation of sleep apnea syndrome with low-field magnetic resonance fluoroscopy. Eur Radiol 1999;9:197-202.

11 Okada T, Fukatsu H, Ishigaki T, et al. Ultra-low-field magnetic resonance imaging in upper airways obstruction in sleep apnea syndrome. Psychiatry Clin Neurosci 1996;50:285-9

12 Gilman S, Low PA, Quinn N, et al. Consensus statement on the diagnosis of multiple system atrophy. J Neurol Sci 1999;163:94-8.

13 Isozaki E, Matsubara S, Hayashida T, et al. Morphometric study of nucleus ambiguus in multiple system atrophy presenting with vocal cord abductor paralysis. Clin Neuropathol 2000;19:213-20.

14 Yasuda T, Senda Y, Koike Y, et al. [Bilateral abductor vocal cord paralysis (Gerhardt syndrome) in the Shy-Drager syndrome]. Rinsho Shinkeigaku 1989;29:1232-6.

15 Williams A, Hanson D, Calne DB. Vocal cord paralysis in the Shy-Drager syndrome. J Neurol Neurosurg Psychiatry 1979;42:151-3.

16 Bannister R, Gibson W, Michaels L, et al. Laryngeal abductor paralysis in multiple system atrophy: a report on three necropsied cases, with observations on the laryngeal muscles and the nuclei ambigui. Brain 1981;104:351-68.

17 Guilleminault C, Tilkian A, Dement WC, et al. The sleep apnea syndrome. Annu Rev Med 1976;27:465-84.

18 Yoshida Y, Mitsumasu T, Miyazaki T, et al. Distribution of motoneurons in the brain stem of monkeys, innervating the larynx. Brain Res Bull 1984;13:413-9.

19 Isono S, Shiba K, Yamaguchi M, et al. Pathogenesis of laryngeal narrowing in patients with multiple system atrophy. J Physiol 2001;536:237-49

20 Kurihara K, Kita K, Hirayama K, et al. [Dysphagia in multiple system atrophy: radiological and manometric study]. Rinsho Shinkeigaku 1993;33:271-7. 